\title{
miR-335 represents an invasion suppressor gene in ovarian cancer by targeting Bcl-w
}

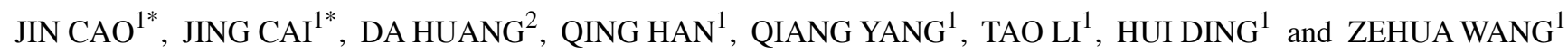 \\ ${ }^{1}$ Department of Obstetrics and Gynecology, and ${ }^{2}$ Department of Clinical Laboratory, Union Hospital, \\ Tongji Medical College, Huazhong University of Science and Technology, Wuhan, Hubei 430022, P.R. China
}

Received February 18, 2013; Accepted April 17, 2013

DOI: 10.3892/or.2013.2482

\begin{abstract}
RNAs (miRNAs) are a class of non-coding small RNAs that bind to target mRNAs, usually resulting in post-transcriptional repression by translational inhibition or target degradation. mRNAs can function as tumor suppressors or oncogenes (also referred to as oncomirs) in human tumors. Although aberrant expression of miR-335 has been reported in ovarian cancer, whether it is an active participant or a mere bystander remains unknown. To clarify its role in ovarian carcinogenesis, we first examined the relative expression of miR-335 in 17 normal ovarian tissues and 4 ovarian cancer cell lines using qPCR. We found that miR-335 was downregulated in the ovarian cancer cell lines relative to normal ovarian epithelium tissues. In vitro, overexpression of miR-335 suppressed cell migration and invasion and resulted in depolymerization of F-actin in ovarian cancer cell lines, but exhibited a negligible effect on cell proliferation. B-cell CLL/lymphoma 2 like 2 (Bcl-w or BCL2L2), a pro-survival member of the Bcl-2 protein family, was identified as a potential target of miR-335 according to the results of bioinformatic analysis, and the expression of Bcl-w and its effector matrix metalloproteinase-2 (MMP-2) was downregulated after transfection with miR-335 mimics. In addition, ectopic Bcl-w could almost fully nullify the effect of miR-335 overexpression on ovarian cancer cell migration and invasion. These findings indicate that the tiny genome product, miR-335, whose lack of expression brings about the abnormal accumulation of Bcl-w and subsequent unchecked cell invasion in ovarian cancer, may help us to understand one of the many steps ovarian cells take
\end{abstract}

Correspondence to: Professor Zehua Wang, Department of Obstetrics and Gynecology, Union Hospital, Tongji Medical College, Huazhong University of Science and Technology, No. 1277 Jiefang Avenue, Wuhan, Hubei 430022, P.R. China

E-mail: zehuawang@163.net

*Contributed equally

Key words: microRNA, ovarian cancer, invasion, B-cell CLL/ lymphoma 2 like 2 on their way toward the acquisition of malignant phenotypes and miR-335 may be a promising predictor of survival.

\section{Introduction}

Due to the insidious nature of ovarian cancer, few patients can be diagnosed before the cancer has spread beyond the ovaries, making ovarian cancer the most lethal gynecological malignancy and the fifth leading cause of cancer-related mortality in women worldwide $(1,2)$. Yet, our understanding of the molecular mechanisms that govern its dissemination remains fragmental.

microRNAs (miRNAs) are small, non-coding RNAs with a length of $\sim 22$ nucleotides, which negatively regulate gene expression by promoting mRNA cleavage or by translational suppression (3). miR-335 has been found to function as an oncogene or tumor-suppressor in a wide variety of cancers (4). It functions as a tumor-suppressor in breast cancer and inhibits metastasis and migration of breast cancer cells (5). Similarly, miR-335 was found to suppress gastric cancer metastasis in vitro and in vivo (6). Lynch et al (7) also found that miR-335 inhibits the invasive and migratory potential of neuroblastoma cells. Interestingly, miR-335 has been reported to be upregulated in glioma (8), as a putative miRNA oncogene in conferring tumorigenic features such as growth and invasion on malignant astrocytoma cell lines in vitro and could be potently required for differentiation of malignant glioma cell lines (9). These findings suggest that the roles of miR-335 vary based on the type of cancer. It will be necessary to determine the expression status of miR-335 in each type of tumor.

Previous profiling studies have associated miR-335 with ovarian cancer (10-12). However, its expression and biological function in ovarian cancer remain unexplored. A search for miR-335 regulatory targets that may be involved in the progression of ovarian cancer using online prediction algorithms has identified Bcl-w, which harbors a putative miR-335 binding site in its 3'-UTR. Bcl-w is an anti-apoptotic member of the Bcl-2 protein family. It is upregulated in tumors such as gastric, colorectal, cervical and breast cancer (13-15). It can promote cell invasion in gastric cancer (13) and inhibit apoptosis in human colorectal cancers (16). These results suggest that the dependency of cancer cells on overexpressed or activated Bcl-w for various tumor-promoting phenotypes, such as sustained survival, proliferation, invasion and metastasis, 
may be different among tissues or cell types. In this study, we demonstrated that miR-335 may act as a metastasis suppressor in ovarian cancer by targeting Bcl-w and MMP-2.

\section{Materials and methods}

Tissue samples. Samples of human normal ovarian tissues were obtained from patients who underwent surgery at Wuhan Union Hospital from 2004 to 2009. The normal tissues were obtained from patients who underwent wedge biopsy of the ovaries, or adnexectomy due to myoma or adenomyosis. After ovary removal, the germinal epithelium was peeled off and subjected to further analysis. After this, 17 normal ovarian tissues were analyzed. Specimen collection and archiving of patient data were performed upon written informed consent and were approved by the ethics committee of the hospital.

Cell culture and transfection. Human epithelial ovarian cancer cell lines, SKOV3, ES2, A2780 and CAOV3, were purchased from the China Center for Type Culture Collection (Wuhan, China) and were propagated in Dulbecco's modified Eagle's medium (DMEM) (Gibco-BRL, Carlsbad, CA, USA) supplemented with $10 \%$ fetal bovine serum (FBS) (Gibco-BRL). For miRNA-335 overexpression, SKOV3 and ES2 cells were transfected with miR-335 mimics or a scrambled sequence as miR-control (GenePharma Co., Ltd., Shanghai, China). For Bcl-w overexpression, SKOV3 cells were transfected with Bcl-w expression constructs, which had been rendered insensitive to miR-335 by deletion of its 3'UTR (GenePharma Co., Ltd.). All transfections were performed using Lipofectamine ${ }^{\mathrm{TM}} 2000$ reagent (Invitrogen Life Technologies, Carlsbad, CA, USA) according to the manufacturer's protocols.

RNA extraction and real-time PCR analysis. Total RNA was extracted with TRIzol reagent (Invitrogen Life Technologies). The expression level of miR-335 was analyzed using the Hairpin-it ${ }^{\mathrm{TM}}$ miRNAs qPCR Quantitation kit (GenePharma, Co. Ltd.), following the manufacturer's instructions. U6 was used as the reference gene for normalization.

For determination of Bcl-w expression levels, reverse transcription was run using RevertAid ${ }^{\mathrm{TM}}$ First Strand cDNA synthesis kit (MBI Fermentas, Flamborough, ON, Canada) according to the supplied protocol. The $25 \mu 1$ polymerase chain reaction (PCR) mixture included 2X SYBR-Green qPCR master mix (Toyobo, Japan), $2.5 \mu \mathrm{l}$ of cDNA template and $0.3 \mu \mathrm{M}$ of each primer. Primers for Bcl-w were as follows: forward, 5'-GAGCCATATAGTTCCTTGGGA-3' and reverse, 5'-TAGAATAAGTGGGGAGTGGGA-3; and for $\beta$-actin, forward, 5'-GCCAACACAGTGCTGTCTGG-3' and reverse, 5'-GCTCAGGAGGAGCAATGATCTTG-3'. $\beta$-actin was used as a reference gene for normalization. The expression of each gene was defined from the threshold cycle $(\mathrm{Ct})$, and relative expression levels were calculated using the $2^{-\Delta \Delta \mathrm{CT}}$ method. All reactions were run in triplicate.

Cell proliferation analysis. Cell proliferation was measured by detecting incorporation of 5-ethynyl-2'-deoxyuridine (EdU) during DNA synthesis using Clik-iT ${ }^{\circledR}$ EdU Imaging kit (Invitrogen Life Technologies) with minor modifications to the manufacturer's protocol. Briefly, cells that had been propagated in a 96-well plate for $48 \mathrm{~h}$ were exposed to $10 \mu \mathrm{M}$ of EdU for $4 \mathrm{~h}$. After EdU exposure, cells were fixed with $4 \%$ formaldehyde and treated with $0.5 \%$ Triton X-100. Proliferative cells were visualized using Click-i ${ }^{\circledR}$ reaction cocktail. Subsequently, cells were counterstained with Hoechst 33342 . All assays were carried out in triplicate.

Migration and invasion assays. To evaluate cell migration and invasion, Transwell cell migration and Matrigel invasion assays were carried out according to a previously reported protocol (17). The results are represented as the means of the cell numbers of 10 randomly selected visual fields (x200 magnification). All assays were performed in triplicate. In corroboration with the Transwell cell migration and Matrigel invasion assays, a wound-healing assay was carried out according to a previously reported protocol (18). Percent migration was calculated by measuring the length and width of the cell-free area. The width was measured at five points along the scratch area and then averaged to obtain an accurate representation of the entire scratch area. Percent migration was determined using the following formula: $[\Delta$ area/area (day 0$)] \times 100$.

F-actin immunofluorescence. SKOV3 cells were transfected with miR-335 mimics or the miR-control, as described above, and plated onto uncoated glass coverslips in a 12-well plate. The cells were fixed with $4 \%$ formaldehyde, permeabilized in $0.1 \%$ Triton X-100 and reacted with phallotoxins (Invitrogen Life Technologies) for $60 \mathrm{~min}$. Subsequently, cell nuclei were counterstained with Hoechst 33342. Images were acquired using an 1X71 digital camera (Olympus, Tokyo, Japan).

Bioinformatics. For miRNA target gene prediction, we used TargetScan release 5.1 online software (http://www.targetscan. org/) and PicTac (http://pictar.mdc-berlin.de/).

Protein extraction and western blot analysis. Total cell extracts were resolved on SDS polyacrylamide gels and blotted onto a Hybond PVDF membrane (GE Healthcare, UK). The following antibodies were used: anti-Bcl-w (1:500 dilution; Cell Signaling Technology, Inc., Beverly, MA, USA), anti- $\beta$-actin (1:1,000 dilution; Santa Cruz Biotechnology, Inc., Santa Cruz, CA, USA), anti-MMP-2 (1:1,000 dilution; Vector Laboratories, Burlingame, CA, USA). The antigen-antibody complexes were visualized using horseradish peroxidaseconjugated secondary antibody (1:5,000 dilution; Santa Cruz Biotechnology, Inc.).

Statistical analysis. Data are presented as means \pm SD from at least 3 separate experiments. Unless otherwise noted, one-way ANOVA was used for comparisons between groups. All statistical analyses were carried out with SPSS 12.0 computer software (SPSS, Inc., Chicago, IL, USA). P $<0.05$ was considered to indicate a statistically significant result.

\section{Results}

miR-335 is downregulated in ovarian cancer cell lines. To determine whether miR-335 plays a role in the tumorigenesis of epithelial ovarian cancer, we examined its expression in 


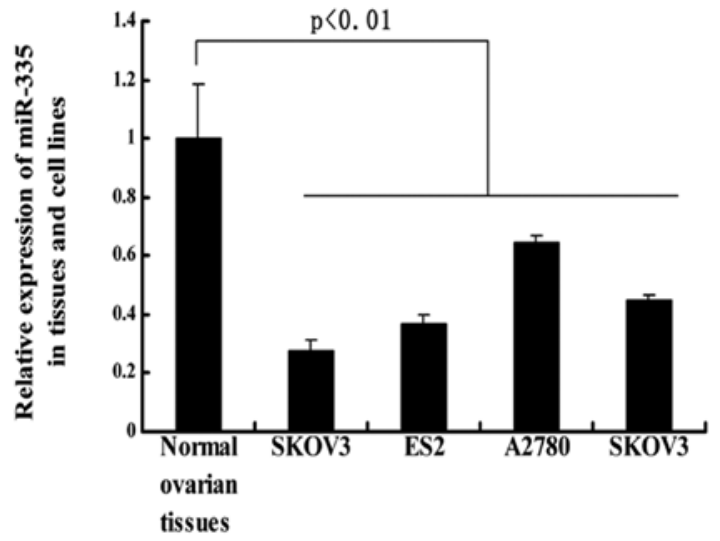

Figure 1. Downregulation of miR-335 expression in ovarian cancer cell lines. Relative expression of miR-335 in normal ovarian tissues and cancer cell lines, SKOV3, ES2, A2780, CAOV3, was measured by quantitative reverse transcription-polymerase chain reaction (qRT-PCR) using U6 as the reference gene. Results represent the mean values. Bars, SD. All reactions were run in triplicate. Relative quantification was performed using the $2^{-\Delta \Delta C T}$ method.

17 normal ovarian tissue samples using stem-loop quantitative reverse transcription-polymerase chain reaction (RT-PCR). We found that miR-335 expression was reduced in ovarian cancer cell lines in comparison with normal ovarian epithelium (Fig. 1).

miR-335 suppresses metastasis-relevant traits in vitro. Given that miR-335 is downregulated in ovarian cancer cell lines, we proceeded to explore its impact on relevant biological behavior of ovarian cancer. Since the expression of miR-335 was most significantly reduced in the SKOV3 and ES2 cell lines, we chose these for further study. Thus, we overexpressed miR-335 in SKOV3 and ES2 cell lines by transiently transfecting them with hsa-miR-335 mimics. Ectopic miR-335 did not affect cell proliferation in vitro (Fig. $2 \mathrm{~A}$ and B), but did reduce the migratory and invasive ability of SKOV3 and ES2 cells (Fig. 2C and D). The effect of miR-335 overexpression on cell migration was further supported by the wound-healing assay (Fig. 2E). Moreover, under a florescence microscope, numerous F-actin bundles were clearly visible in SKOV3 cells transfected with the miR-335 mimics, while control cells contained markedly fewer, finer and shorter bundles and exhibited an elongated morphology with lamellipodia (Fig. 2F), suggesting miR-335 exerts anti-migratory activity in ovarian cancer cells at least partly by inhibiting actin polymerization.

miR-335 suppresses the invasiveness of ovarian cancer by targeting Bcl-w and Bcl-w effector, MMP-2. For miRNA target gene prediction, we used TargetScan release 5.1 online software (http://www.targetscan.org/, Whitehead Institute for Biomedical Research, Cambridge, MA, USA). Among a total of 146 genes that was potentially targeted by miR-335, we found that Bcl-w may contribute to the metastasis of ovarian cancer. To establish that miR-335 targets Bcl-w in ovarian cancer, we overexpressed miR-335 in SKOV3 and ES2 cells. We found that ectopic miR-335 significantly repressed Bcl-w protein levels while no difference was detected between the miR-control and untreated cells. Moreover, a concomitant repression of the
Bcl-w effector, MMP-2, was also observed (Fig. 3A and B). These observations demonstrated that miR-335 targets Bcl-w in ovarian cancer. In spite of these findings, no reduction in Bcl-w was observed at the mRNA level when miR-335 was modulated in SKOV3 and ES2 cells (Fig. 3C and D).

Ectopic Bcl-w reverses miR-335-mediated invasion defects. To establish that the ability of miR-335 to impede cell invasion is mainly mediated by the downregulation of $\mathrm{Bcl}-\mathrm{w}$, we concomitantly transfected SKOV3 and ES2 cells with 335 mimics and Bcl-w expression constructs. In these cells, ectopic Bcl-w reversed the miR-335-imposed invasion defects (Fig. 4C and D). Under the same conditions, we observed a significant increase in MMP-2 (Fig. 4A and B).

\section{Discussion}

miRNAs can modulate a wide variety of biological processes. In the present report, we demonstrated that a single human miRNA, miR-335, is endowed with the ability to repress the pro-metastatic target, Bcl-w, and thereby to inhibit invasion in ovarian cancer.

To date, only a limited number of miRNAs with pro-metastatic or anti-metastatic functions have been identified in ovarian cancer (19-23), miR-34 has been reported to be endowed with the ability to mitigate invasion (19). miR-21 has been categorized as a pro-metastatic gene (22). However, its specific contributions to metastasis are not easily discerned as they have significant effects on cell proliferation at the same time. In contrast, miR-335 obstructs invasion without exerting confounding influences on cell proliferation. As such, it may aptly be categorized as an 'exclusive invasion suppressor'.

Bcl-w is an anti-apoptotic member of the Bcl-2 protein family. It suppresses apoptosis by direct interaction with pro-apoptotic members and by blocking their apoptotic activities (24-27), and previous evidence suggests that the expression of Bcl-w is associated with infiltrative morphotypes (14). Recently, reporter gene assays have validated its role as an effector of miR-335 (6). Although mutations in the anti-apoptotic Bcl-2 family members (Bcl-xL, Mcl-1, Bcl-w and A1) have not been identified as a cause of tumors, high expression of these proteins can contribute to carcinogenesis in cooperation with other proto-oncogenes (28). Bcl-w can promote cell invasion by activating the PI3K-Akt-Sp1-MMP-2 pathway (13) and blocking the invasion suppression action of Bax (29). In our research, we demonstrated that miR-335 supressed the invasiveness of ovarian cancer by targeting Bcl-w and Bcl-w effector, MMP-2.

In spite of this, we found that miR-335-mediated defects in invasion could not be fully mitigated by ectopic Bcl-w, and that miR-335 affected actin polymerization, an important step in EMT. Thus, the possibility that miR-335-mediated defects in invasion are achieved by recruiting a cohort of effectors cannot formally be excluded, again highlighting the pleiotropic action of miRNAs.

miRNAs effect gene silencing via both translational repression or mRNA degradation pathways. When an miRNA and its cognate mRNA interact with perfect complementarity, the target mRNA is directly cleaved, resulting in the reduction in the abundance of the target mRNA. However, in animals, 

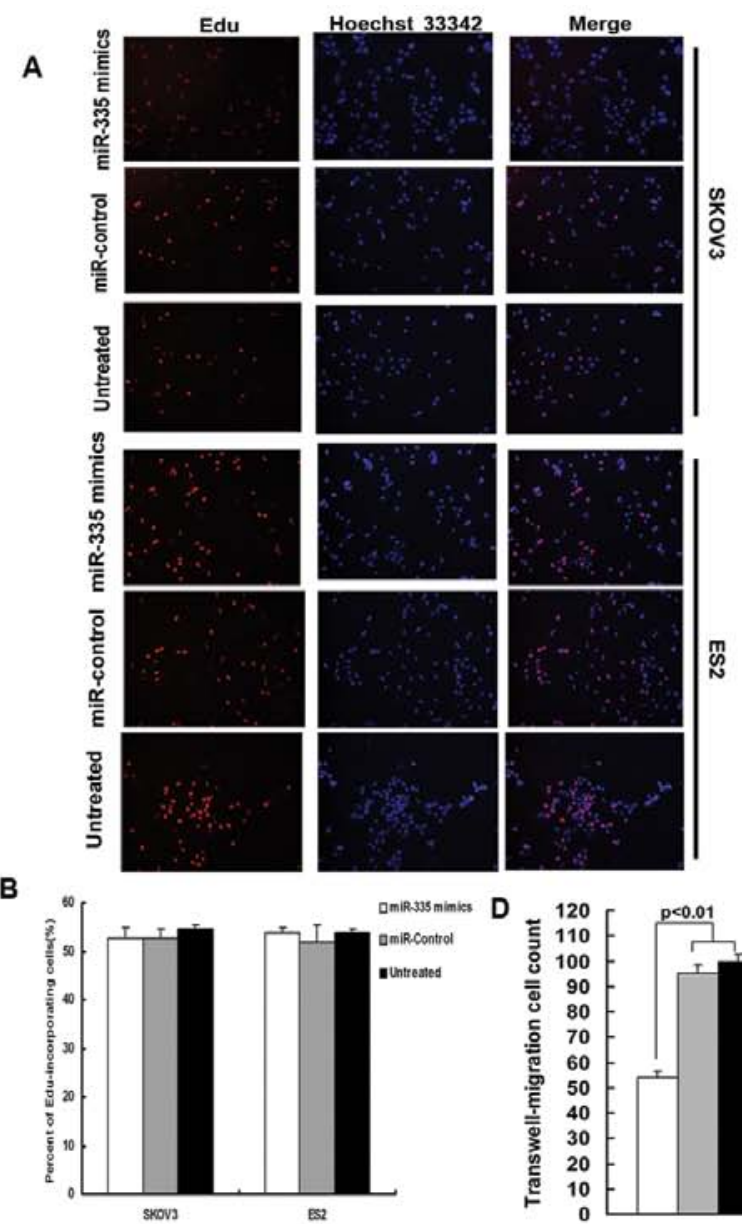

C

\section{miR-335 mimics miR-Control Untreated}
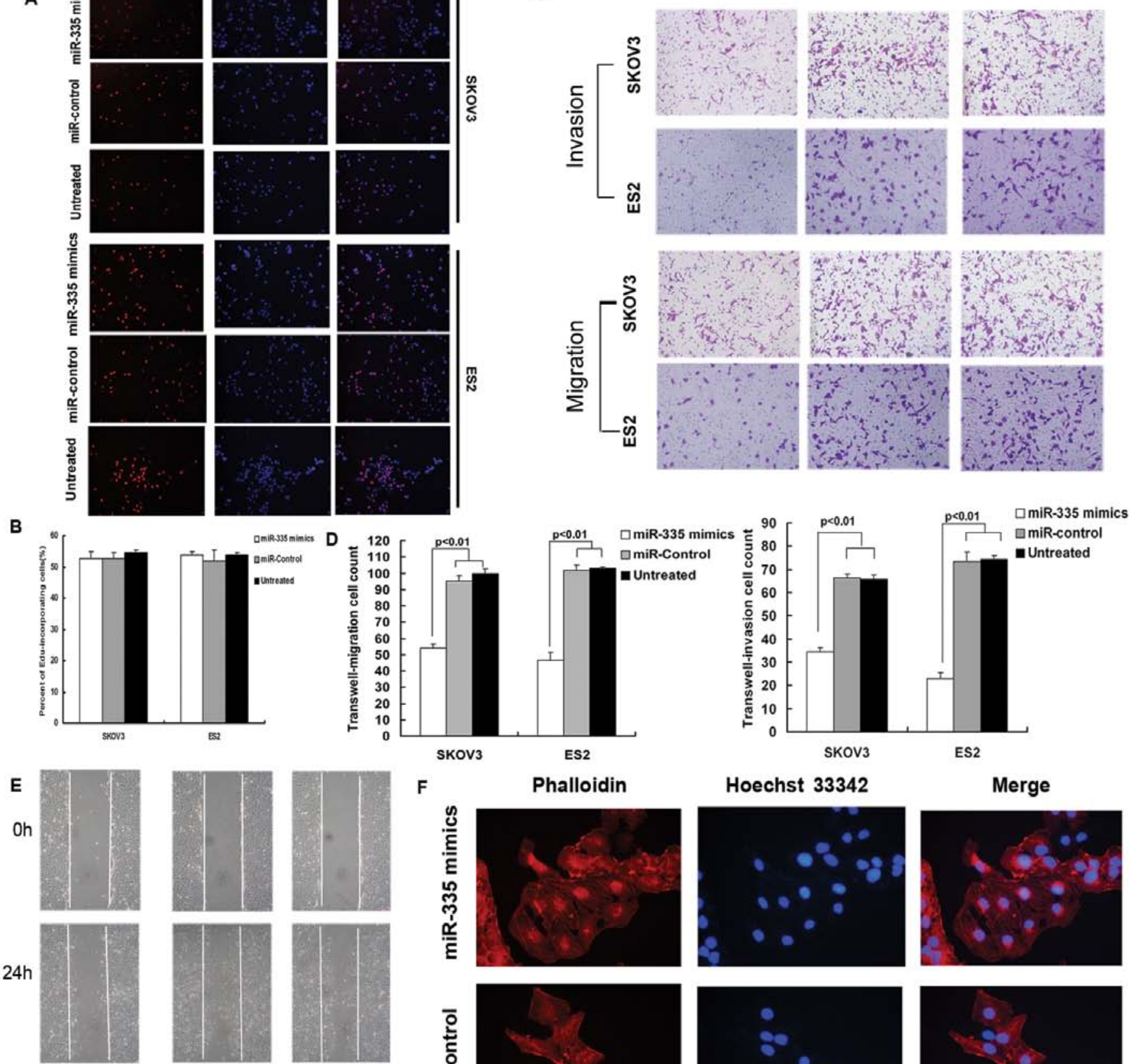

miR-335 mimics miR-control

Untreated

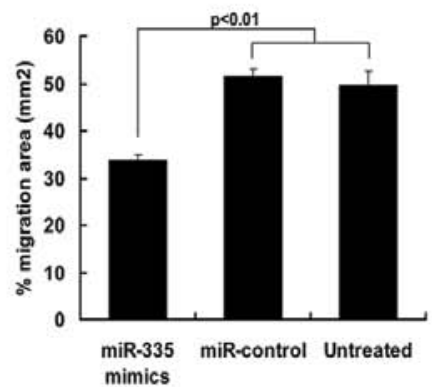

F

Phalloidin
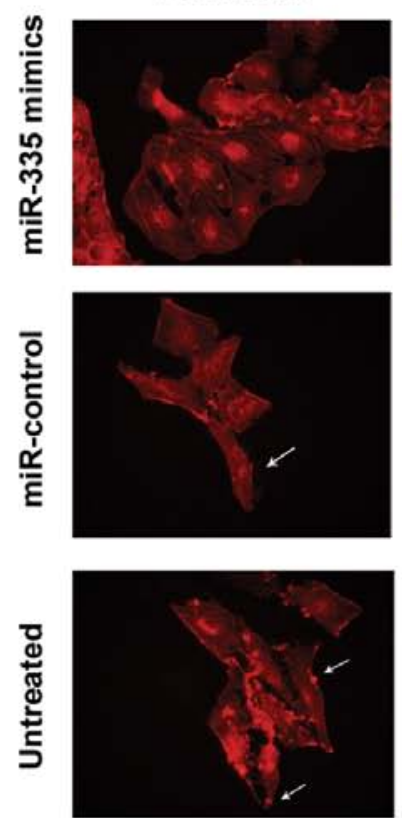

\section{Hoechst 33342}
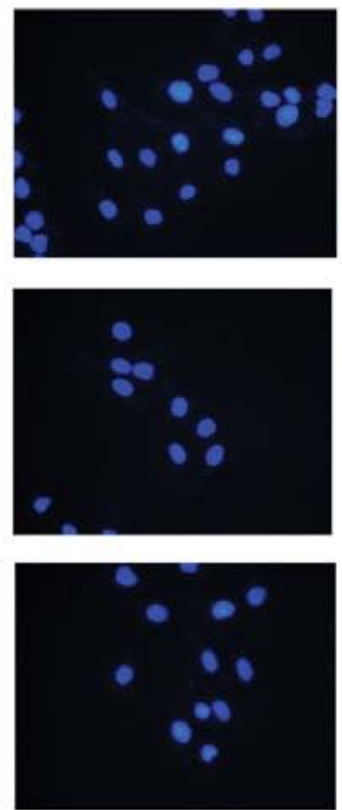

Merge
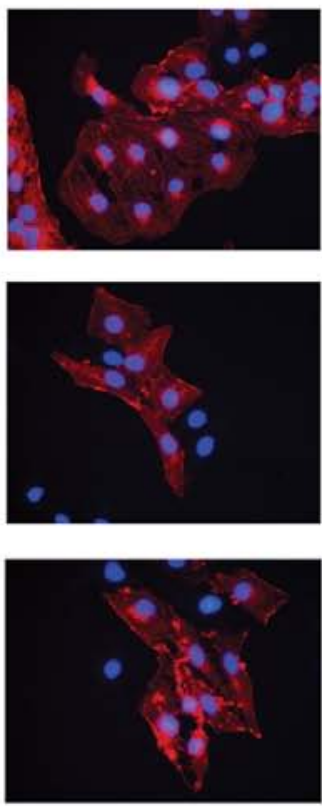

Figure 2. miR-335 suppresses metastasis-relevant traits in vitro. SKOV3 and ES2 cells were transfected with miR-335 mimics or the miR-control. (A) Representative photomicrographs of EdU results for SKOV3 and ES2 cells were captured at x200 magnification. (B) Mean of EdU triplicate experiments for SKOV3 and ES2; bars, SD. (C) The indicated cell transfectants were seeded onto either uncoated or Matrigel-coated polycarbonate filters to analyze their migratory and invasive potentials. Cells that migrated through the filters were stained and counted under a microscope at x200 magnification. (D) Mean values of Transwell triplicate experiments for SKOV3 and ES2 cells; bars, SD. (E) SKOV3 cells were seeded in 6-well plates and wounded on the next day. Images were captured under a microscope at x 200 magnification at 0 and $24 \mathrm{~h}$ after the wounding. (F) Staining of the F-actin cytoskeleton with phalloidin revealed extensive pseudopodia and lamellipodia (arrows) in SKOV3 cells. The images were captured under x400 magnification. Nuclei were stained with Hoechst 33342. 
A
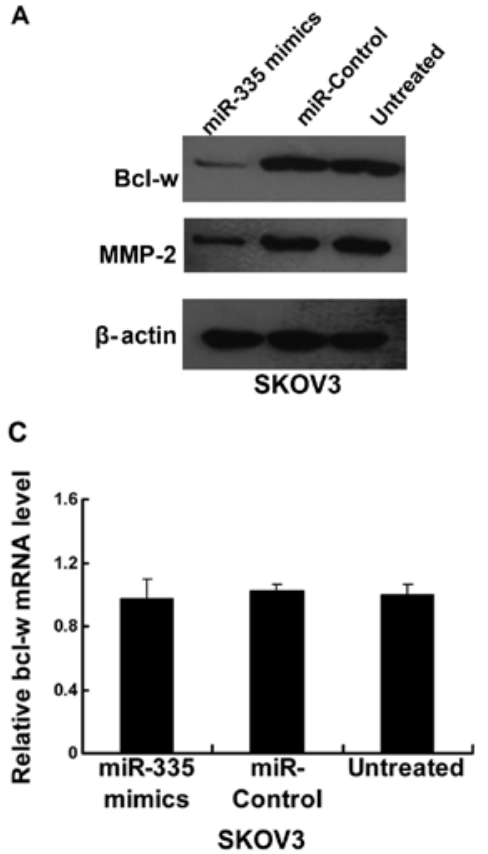

B
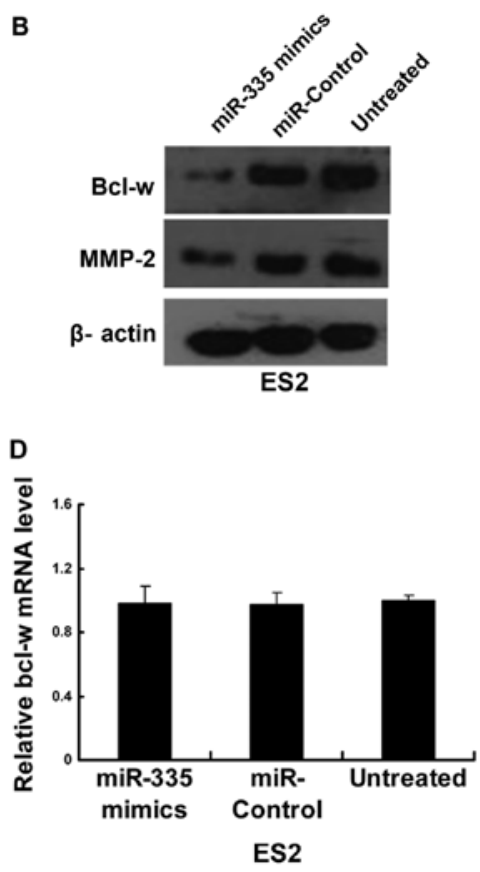

Figure 3. miR-335 inhibits cell migration and invasion via Bcl-w by decreasing MMP-2 in SKOV3 and ES2 cells. (A and B) The mRNA levels in the indicated SKOV3 and ES2 transfectants were compared using quantitative reverse transcription-polymerase chain reaction (qRT-PCR). (C and D) Cellular levels of Bcl-w and MMP-2 in the indicated SKOV3 and ES2 cell transfectants were compared by western blotting using $\beta$-actin as a loading control.

A

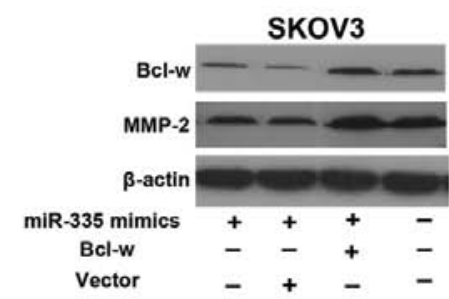

B

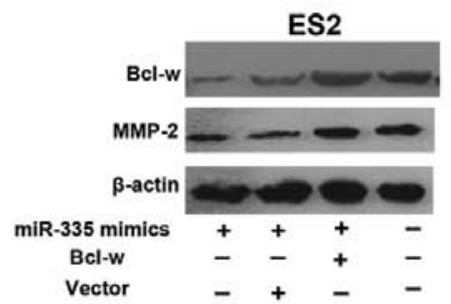

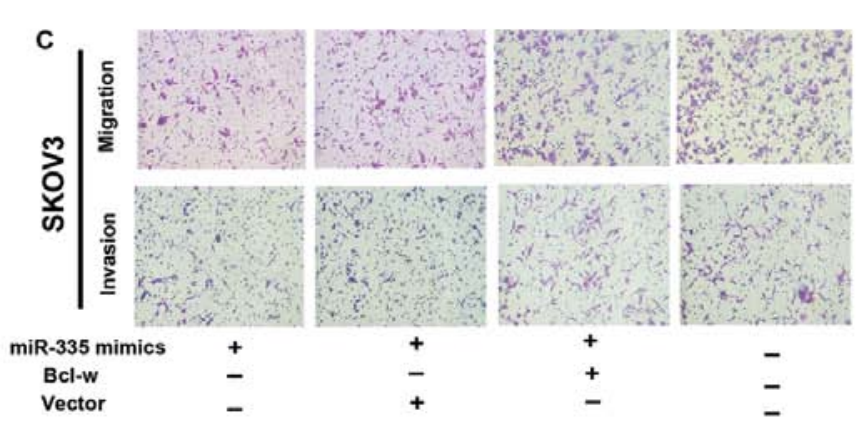

D
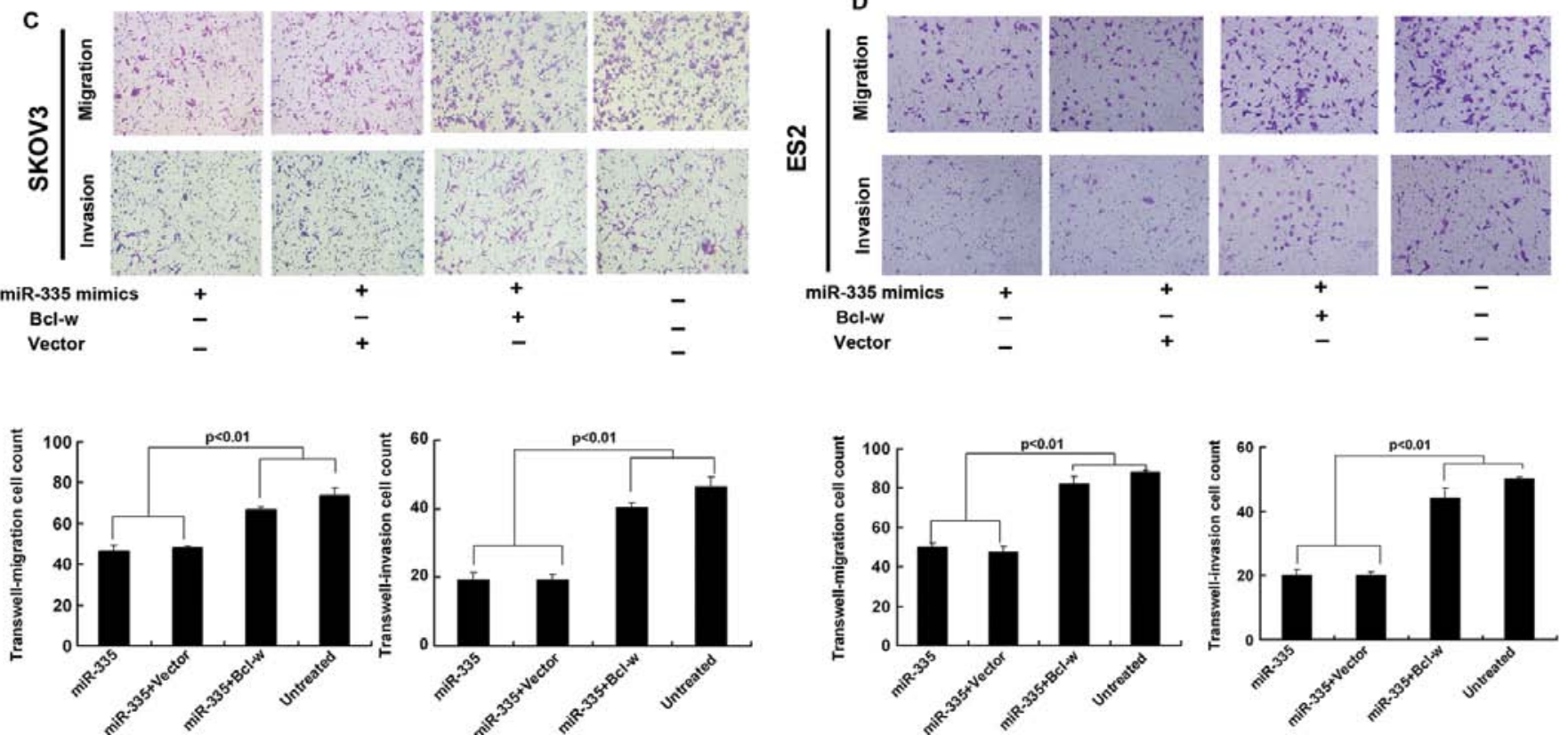

Figure 4. Ectopic Bcl-w offsets miR-335-mediated invasion. Bcl-w expression vectors were constructed and introduced into SKOV3 and ES2 miR-335 mimic transfectants. (A and B) Cellular levels of Bcl-w and MMP-2 in the indicated SKOV3 and ES2 cell transfectants were compared by western blotting using $\beta$-actin as a loading control. (C and D) Top, the migratory and invasive potentials of the co-transfectants were compared with the Bcl-w transfectants and miR-control. Bottom, columns, mean of triplicate experiments; bars, SD. 
miRNAs generally interact with target mRNAs with only partial complementarity. This mode of recognition results in translational repression of the target mRNA with much smaller effects on the level of target mRNA abundance $(6,12,15,30)$. In our study, it was found that ectopic miR-335 significantly reduced Bcl-w expression at the protein level. However, no corresponding decrease in Bcl-w expression was observed at the mRNA level. In fact, such a finding is not unprecedented (19) and is consistent with the notion that mammalian cells favor translational repression.

In summary, our results suggest that decreased miR-335 facilitates ovarian cancer invasion via the $\mathrm{Bcl}-\mathrm{w}$ pathway. To our knowledge, this represents the first observation of reduced miR-335 expression in human ovarian cancer. Moreover, as metastases are responsible for patient mortality in ovarian cancer, the ability of miR-335 to impede invasion may prove to be clinically useful.

\section{Acknowledgements}

We thank the Department of Obstetrics and Gynecology, Union Hospital, Wuhan, Hubei, China, for providing the tissue samples. This study was supported by the Scientific Research Foundation for the Returned Overseas Chinese Scholars, the State Education Ministry.

\section{References}

1. Jemal A, Tiwari RC, Murray T, et al: Cancer statistics, 2004. CA Cancer J Clin 54: 8-29, 2004.

2. Moss $\mathrm{C}$ and Kaye SB: Ovarian cancer: progress and continuing controversies in management. Eur J Cancer 38: 1701-1707, 2002

3. Esquela-Kerscher A and Slack FJ: Oncomirs - microRNAs with a role in cancer. Nat Rev Cancer 6: 259-269, 2006.

4. Kong YW, Ferland-McCollough D, Jackson TJ and Bushell M: microRNAs in cancer management. Lancet Oncol 13: e249-e258, 2012.

5. Tavazoie SF, Alarcón C, Oskarsson T, et al: Endogenous human microRNAs that suppress breast cancer metastasis. Nature 451 147-152, 2008.

6. Xu Y,Zhao F, Wang Z, et al: microRNA-335 acts as a metastasis suppressor in gastric cancer by targeting Bcl-w and specificity protein 1. Oncogene 31: 1398-1407, 2012.

7. Lynch J, Fay J, Meehan M, et al: miRNA-335 suppresses neuroblastoma cell invasiveness by direct targeting of multiple genes from the non-canonical TGF- $\beta$ signalling pathway. Carcinogenesis 33: 976-985, 2012.

8. Schmitz KJ, Helwig J, Bertram S, et al: Differential expression of microRNA-675, microRNA-139-3p and microRNA-335 in benign and malignant adrenocortical tumours. J Clin Pathol 64 : 529-535, 2011

9. Shu M, Zhou Y, Zhu W, et al: microRNA 335 is required for differentiation of malignant glioma cells induced by activation of cAMP/protein kinase A pathway. Mol Pharmacol 81: 292-298, 2012.

10. Dahiya N, Sherman-Baust CA, Wang TL, et al: microRNA expression and identification of putative miRNA targets in ovarian cancer. PLoS One 3: e2436, 2008.
11. Sorrentino A, Liu CG, Addario A, Peschle C, Scambia G and Ferlini C: Role of microRNAs in drug-resistant ovarian cancer cells. Gynecol Oncol 111: 478-486, 2008

12. Wyman SK, Parkin RK, Mitchell PS, et al: Repertoire of microRNAs in epithelial ovarian cancer as determined by next generation sequencing of small RNA cDNA libraries. PLoS One 4: e5311, 2009.

13. Bae IH, Park MJ, Yoon SH, et al: Bcl-w promotes gastric cancer cell invasion by inducing matrix metalloproteinase-2 expression via phosphoinositide 3-kinase, Akt, and Sp1. Cancer Res 66: 4991-4995, 2006

14. Lee HW, Lee SS, Lee SJ and Um HD: Bcl-w is expressed in a majority of infiltrative gastric adenocarcinomas and suppresses the cancer cell death by blocking stress-activated protein kinase/c-Jun $\mathrm{NH}_{2}$-terminal kinase activation. Cancer Res 63: 1093-1100, 2003.

15. Wilson JW, Nostro MC, Balzi M, et al: Bcl-w expression in colorectal adenocarcinoma. Br J Cancer 82: 178-185, 2000.

16. Kitamura S, Kondo S, Shinomura Y, et al: Met/HGF receptor modulates bcl-w expression and inhibits apoptosis in human colorectal cancers. Br J Cancer 83: 668-673, 2000.

17. Lü MH, Li CZ, Hu CJ, et al: microRNA-27b suppresses mouse MSC migration to the liver by targeting SDF-1 $\alpha$ in vitro. Biochem Biophys Res Commun 421: 389-395, 2012.

18. Moore LD, Isayeva T, Siegal GP and Ponnazhagan S: Silencing of transforming growth factor- $\beta 1$ in situ by RNA interference for breast cancer: implications for proliferation and migration in vitro and metastasis in vivo. Clin Cancer Res 14: 4961-4970, 2008.

19. Corney DC, Hwang CI, Matoso A, et al: Frequent downregulation of miR-34 family in human ovarian cancers. Clin Cancer Res 16: 1119-1128, 2010.

20. Fan X, Liu Y, Jiang J, et al: miR-20a promotes proliferation and invasion by targeting APP in human ovarian cancer cells. Acta Biochim Biophys Sin 42: 318-324, 2010.

21. Li J, Liang S, Yu H, Zhang J, Ma D and Lu X: An inhibitory effect of miR-22 on cell migration and invasion in ovarian cancer. Gynecol Oncol 119: 543-548, 2010.

22. Lou Y, Yang X, Wang F, Cui Z and Huang Y: MicroRNA-21 promotes the cell proliferation, invasion and migration abilities in ovarian epithelial carcinomas through inhibiting the expression of PTEN protein. Int J Mol Med 26: 819-827, 2010.

23. Tsuda N, Kawano K, Efferson CL and Ioannides CG: Synthetic microRNA and double-stranded RNA targeting the 3'-untranslated region of HER-2/neu mRNA inhibit HER-2 protein expression in ovarian cancer cells. Int J Oncol 27: 1299-1306, 2005.

24. Antonsson B: Bax and other pro-apoptotic Bcl-2 family 'killer-proteins' and their victim the mitochondrion. Cell Tissue Res 306: 347-361, 2001.

25. Bae IH, Yoon SH, Lee SB, Park JK, Ho JN and Um HD: Signaling components involved in Bcl-w-induced migration of gastric cancer cells. Cancer Lett 277: 22-28, 2009.

26. Gross A, McDonnell JM and Korsmeyer SJ: BCL-2 family members and the mitochondria in apoptosis. Genes Dev 13 1899-1911, 1999.

27. Uren RT, Dewson G, Chen L, et al: Mitochondrial permeabilization relies on BH3 ligands engaging multiple prosurvival Bcl-2 relatives, not Bak. J Cell Biol 177: 277-287, 2007.

28. Zhivotovsky B and Orrenius S: Carcinogenesis and apoptosis: paradigms and paradoxes. Carcinogenesis 27: 1939-1945, 2006.

29. Kim EM, Kim J, Park JK, et al: Bcl-w promotes cell invasion by blocking the invasion-suppressing action of Bax. Cell Signal 24: 1163-1172, 2012.

30. Wightman B, Ha I and Ruvkun G: Posttranscriptional regulation of the heterochronic gene lin-14 by lin- 4 mediates temporal pattern formation in C.elegans. Cell 75: 855-862, 1993. 